\title{
Perfil clínico de mulheres com incontinência urinária de esforço em centro de referência
}

\section{Clinical profile of women with stress urinary incontinence in a reference center}

\author{
Rafael Andrade Alves ${ }^{1}$ \\ Mohana Machado ${ }^{2}$ (B) \\ Thais Moura ${ }^{3}$ (1)
}

Cristina Aires Brasil 4

Amanda Queiroz Lemos 5

Patricia Lordelo 6

\begin{abstract}
1,4Escola Bahiana de Medicina e Saúde Pública (Salvador). Bahia, Brasil. rafaelalvescirurgia@gmail.com, tinaabrasil@hotmail.com
2,3Universidade Salvador (Salvador). Bahia, Brasil. machadomohana@gmail.com, thais-moura95@hotmail.com 5União Metropolitana de Educação e Cultura (Salvador). Bahia, Brasil. aq.lemos@hotmail.com ${ }^{6}$ Autora para correspondência. Escola Bahiana de Medicina e Saúde Pública (Salvador). Bahia, Brasil. pvslordelo@hotmail.com
\end{abstract}

RESUMO | INTRODUÇÃo: A incontinência urinária é definida como qualquer perda involuntária de urina. É um sério problema de saúde pública e as mulheres são as mais afetadas e apresentam como fatores de risco o envelhecimento, mais de duas gestações, parto vaginal com episiotomia, entre outros. Embora a IU não seja uma condição de vida ameaçadora, pode levar a situações com repercussões a nível social e pessoal, com influência na qualidade de vida. OBJETIVO: Descrever a frequência da IUE em um centro especializado na cidade de Salvador, assim como apontar as características clínicas, fatores de risco e comorbidades associadas à IUE feminina. MATERIAIS E MÉTODOS: Estudo transversal descritivo, a partir da análise de dados de prontuários de mulheres portadoras de incontinência urinária de esforço, incluídos dados sociodemográgicos, fatores de risco, comorbidades associadas, queixas clínicas e dados objetivos de Pad Test e Diário Miccional. RESULTADOS: Foram incluídas 28 mulheres com idade média de 48,9 anos $( \pm 7,7)$, de raça parda $(46,2 \%)$, com ensino médio completo (40\%), casadas (52\%), trabalhadoras do lar (32,2\%), IMC médio 26,2 ( $\pm 4,9)$. A comorbidade associada mais predominante foi obesidade $(28,6 \%)$, o fator de risco dominante foi o consumo de café (70\%). A queixa clínica mais prevalente foi perda ao tossir (96,3\%). Quando analisado Pad test, notado maior prevalência de perda leve $(57,14 \%)$, seguido por $(39,29 \%)$ de perda moderada e perda grave $(3,57 \%)$. CONCLUSÃO: Mulheres de meia idade, pardas, menopausadas, obesas, hipertensas, multíparas, que realizaram parto vaginal com episiotomia, constipadas e que ingerem cafeína são mais propensas a desenvolver a incontinência urinária de esforço. Houve uma maior prevalência de incontinência urinária leve.

PALAVRAS-CHAVE: Incontinência urinária de esforço. Mulheres. Transtornos urinários.
ABSTRACT | INTRODUCTION: Urinary incontinence is defined as any involuntary loss of urine. It is a serious public health problem, and women are the most affected and present aging as risk factors, more than two pregnancies, vaginal delivery with episiotomy, among others. Although $\mathrm{UI}$ is not a threatening life condition, it can lead to social and personal repercussions, influencing the quality of life. OBJECTIVE: Describe the frequency of SUI in a specialized center in the city of Salvador, as well as point out the clinical characteristics, risk factors, and comorbidities associated with female SUI. MATERIALS AND METHODS: Descriptive cross-sectional study, based on data analysis of medical records of women with stress urinary incontinence, including sociodemographic data, risk factors, associated comorbidities, clinical complaints, and objective data from Pad Test and Diary Diary. RESULTS: Twenty-eight women with an average age of 48.9 years $( \pm 7.7)$, brown race $(46.2 \%)$, complete high school $(40 \%)$, married $(52 \%)$, housewives (32.2\%), mean BMI $26.2( \pm 4.9)$. The most prevalent associated comorbidity was obesity (28.6\%); the dominant risk factor was coffee consumption (70\%). The most prevalent clinical complaint was cough loss (96.3\%). When analyzed Pad test noted a higher prevalence of mild loss (57.14\%), followed by (39.29\%) moderate loss and severe loss (3.57\%). CONCLUSION: Middle-aged, mulatto, menopausal, obese, hypertensive, multiparous women who had a vaginal delivery with episiotomy, constipation, and caffeine intake are more likely to develop stress urinary incontinence. There was a higher prevalence of mild urinary incontinence.

KEYWORDS: Stress urinary incontinence. Women. Urinary disorders. 


\section{Introdução}

A International Continence Society (ICS) define a incontinência urinária (IU) como qualquer perda involuntária de urina que se apresenta de forma muito diversificada, desde perdas muito rápidas e ocasionais, a perdas mais graves e regulares, na ausência de infecção do trato urinário (ITU) ou outra patologia que tivesse relação com esse sintoma. ${ }^{1}$ É um sério problema de saúde pública ${ }^{1}$ e as mulheres são as

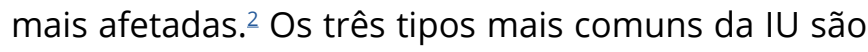
a incontinência de esforço (IUE), incontinência de urgência (IUU) e incontinência mista (IUM). 를

Os dados epidemiológicos da IU não são facilmente encontrados, talvez pelo estereótipo negativo que dificulta a adesão ao tratamento da disfunção. ${ }^{-3}$ Todavia, a incontinência urinária de esforço é relatada como a mais prevalente. ${ }^{3-5}$ Embora a IU não seja uma condição de vida ameaçadora, pode levar a situações com repercussões a nível social e pessoal, com influência na qualidade de vida, levando à marginalização do convívio social, frustrações psicossociais, institucionalização precoce. ${ }^{-6-8}$ Estudos demonstram impacto negativo na autoestima das mulheres com IU, que muitas vezes precisam fazer uso contínuo de absorventes, dificultando a realização de atividades de vida diárias, prejudicando a qualidade de vida e vivendo situações constrangedoras perante à sociedade. ${ }^{9}$

Os fatores de risco para IUE apresentados na literatura são envelhecimento, mais de duas gestações, parto vaginal com episiotomia, herança étnica, tabagismo, obesidade, ingestão de cafeína, constipação e menopausa. ${ }^{10,11}$ Os principais sintomas relatados por mulheres que apresentam IUE são a perda urinaria ao tossir, espirrar, caminhar, exercitar, pular, ou seja, realizar ação que demande algum tipo de esforço.., 12 Isso ocorre quando existe uma pressão sobre a bexiga e os músculos do assoalho pélvico estão enfraquecidos, não conseguindo manter o fechamento do esfíncter. 6,12

O conhecimento dos fatores de risco e dados clínicos obtidos através de uma anamnese detalhada, bem como os dados objetivos de exames como Pad Test e diário miccional, são importantes para determinar um diagnóstico e optar por intervenção precoce da equipe de saúde. ${ }^{13,14} \mathrm{~A}$ literatura apresenta escassez de estudos que descrevam o perfil e as queixas frequentes em mulheres portadoras de IUE, portanto, este estudo tem como objetivo descrever a frequência da IUE em um centro especializado na cidade de Salvador, assim como apontar as características clínicas, fatores de risco e comorbidades associadas à IUE feminina.

\section{Materiais e métodos}

Foi realizado um estudo transversal descritivo realizado no Centro de Atenção ao Assoalho Pélvico (AAP), pertencente à Escola Bahiana de Medicina e Saúde Pública (EBMSP), em Salvador, Bahia. Esta pesquisa foi aprovada pelo Comitê de Ética em Pesquisa da EBMSP) (CAAE - 35038914.3.0000.5544), todas as participantes assinaram o Termo de Consentimento Livre e Esclarecido (TCLE).

Foram incluídas mulheres que apresentavam diagnóstico clínico de incontinência urinária de esforço, com idade entre 18 anos e 65 anos, acompanhadas pelo serviço entre os anos de 2014 a 2018. Foram excluídas aquelas que relataram doença psiquiátrica e/ ou neurológicas, presença de prolapsos, perda urinária por urgência e gestantes.

Após o consentimento das participantes, foi aplicado um questionário de dados sóciodemográficos e características clínicas, especialmente desenvolvido para este fim, com dados sociodemográficos (idade, raça/cor, escolaridade, religião, estado civil, profissão, IMC), comorbidades associadas (obesidade, hipertensão, diabetes mellitus, doenças vasculares, constipação, enurese na infância), prática de atividade física, fatores de riscos (gestação, parto, aborto, tipo de parto, alimentos irritativos, tipos de alimentos). Além de sintomas clínicos como disúria, urgência, sensação de resíduo, enurese, menopausa, tipo de esforço, frequência de perda ao esforço, frequência miccional diurna e noturna e a ingesta hídrica.

As participantes foram instruídas a realizar um diário miccional (DM) em domicílio, durante três dias, para registro da ingesta hídrica, volume urinário médio, frequência e intervalo miccional e o número de episódios de incontinência. 


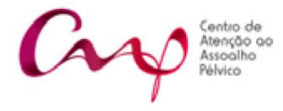

DLÁRIO MICCIONAL

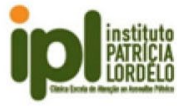

Paciente:
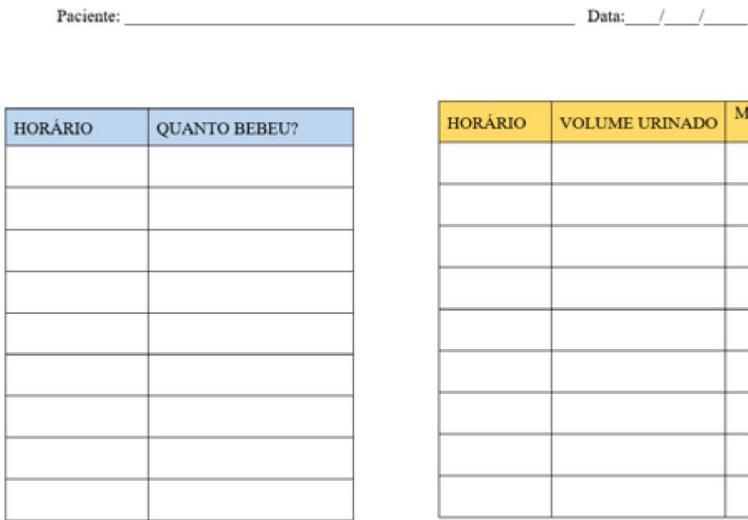

\begin{tabular}{|l|l|l|l|}
\hline HORARIO & VOLUME URINADO & $\begin{array}{c}\text { MOLHOU A CALCINHA } \\
\text { OU CUECA? }\end{array}$ & OBSERVAÇÕES \\
\hline & & & \\
\hline & & & \\
\hline & & & \\
\hline & & & \\
\hline & & & \\
\hline & & & \\
\hline & & & \\
\hline & & & \\
\hline & & & \\
\hline
\end{tabular}

Após a realização do DM, as participantes realizaram o Pad Test de uma hora para quantificar a perda urinária em gramas. Inicialmente, o absorvente foi colocado em um saco plástico fechado e pesado em uma balança eletrônica de precisão (Denver Instrument ${ }^{\circledR}$ ). Em seguida, a participante é instruída a colocar o absorvente, ingerir $500 \mathrm{ml}$ de água em no máximo 15 minutos e permanecer em repouso por mais 15 minutos; esta fase representa 30 minutos do teste. Na outra metade de uma hora, foi solicitado a execução de determinadas atividade padronizadas simulando atividades da vida diária (subir e descer uma escada, sentar-se e levantar dez vezes, tossir dez vezes, pegar objetos no chão cinco vezes, correr no mesmo lugar por um minuto e lavar as mãos em água corrente por um minuto). A perda urinária foi categorizada em ausência $<1$, leve 1-10, moderada 10-50 e grave $>50 . \frac{15}{}$

Para elaboração do banco de dados e análise, utilizado o software Statistical Package for Social Sciences (SPSS), versão 14.0 para Windows. A normalidade das variáveis numéricas foi verificada através do teste KolmogorovSmirnov, estatística descritiva e análise gráfica. Os resultados foram apresentados por meio de tabelas e gráficos. As variáveis categóricas, expressas em valores absolutos e percentuais - $\mathrm{n}$ (\%). As variáveis contínuas com distribuição normal foram expressas em média e desvio padrão (DP) e aquelas com distribuição assimétrica, em mediana e intervalo interquartil (IQ).

\section{Resultados}

Figura 2. Fluxograma da pesquisa. Salvador, 2018

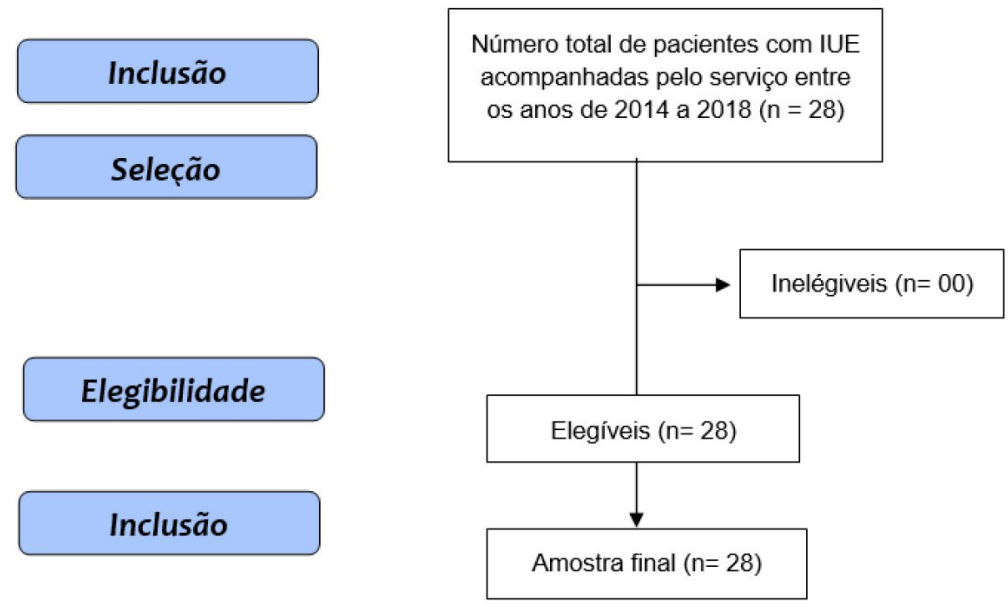


Foram analisadas 28 mulheres com IUE. Quanto aos dados sociodemográficos, encontrada maior prevalência em mulheres de meia-idade 48,9 anos $( \pm 7,7)$, de raça parda $(46,2 \%)$, com ensino médio completo $(40 \%)$, casadas (52\%), católicas (60\%), trabalhadoras do lar $(32,2 \%)$, com IMC médio $26,2( \pm 4,9)$. A comorbidade associada mais predominante foi obesidade $(28,6 \%)$ seguida de enurese na infância $(25,9 \%)$, hipertensão arterial $(23,8 \%)$ e constipação (23,8\%), como mostrado na Tabela 1.

Tabela 1. Descrição das variáveis sociodemográficas das 28 participantes com queixa clínica de incontinência urinária de esforço. Salvador, 2018 (continua)

\begin{tabular}{|c|c|}
\hline Variáveis & Resultados \\
\hline Idade* & $48,9 \pm 7,7$ \\
\hline IMC* & $26,2 \pm 4,9$ \\
\hline Estado civil & $n(\%)$ \\
\hline Casada & $13(52,0)$ \\
\hline Solteira & $08(32,0)$ \\
\hline Viúva & $04(16,0)$ \\
\hline \multicolumn{2}{|l|}{ Escolaridade } \\
\hline Superior completo & $04(16,0)$ \\
\hline Superior incompleto & $02(8,0)$ \\
\hline Médio completo & $10(40,0)$ \\
\hline Médio incompleto & $02(8,0)$ \\
\hline Fundamental completo & $06(24,0)$ \\
\hline Fundamental incompleto & $01(4,0)$ \\
\hline \multicolumn{2}{|l|}{ Ocupação } \\
\hline Aposentada & $03(10,7)$ \\
\hline Atendente & $01(3,6)$ \\
\hline Autônomo & $01(3,6)$ \\
\hline Auxiliar financeiro & $01(3,6)$ \\
\hline Auxiliar de serviços & $01(3,6)$ \\
\hline Costureira & $01(3,6)$ \\
\hline Desempregada & $01(3,6)$ \\
\hline Doméstica & $03(10,7)$ \\
\hline Dona de casa & $06(21,5)$ \\
\hline Estudante & $02(7,1)$ \\
\hline Gerente & $01(3,6)$ \\
\hline Marisqueira & $01(3,6)$ \\
\hline Nutricionista & $01(3,6)$ \\
\hline Professora & $01(3,6)$ \\
\hline Vendas & $01(3,6)$ \\
\hline \multicolumn{2}{|l|}{ Religião } \\
\hline Católico & $15(60,0)$ \\
\hline Evangélico & $05(20,0)$ \\
\hline Sem religião definida & $04(16,0)$ \\
\hline Adventista & $01(4,0)$ \\
\hline
\end{tabular}


Tabela 1. Descrição das variáveis sociodemográficas das 28 participantes com queixa clínica de incontinência urinária de esforço. Salvador, 2018 (conclusão)

\begin{tabular}{lc}
\hline Variáveis & Resultados \\
\hline Raça & $12(46,2)$ \\
Parda & $09(34,6)$ \\
Preta & $05(19,2)$ \\
Branca & \\
\hline Comorbidades associadas & $06(28,6)$ \\
Obesidade & $05(23,8)$ \\
Hipertensão Arterial & $01(4,8)$ \\
Diabetes mellitus & $04(19,0)$ \\
Doenças vasculares & $05(23,8)$ \\
Constipação & $07(25,9)$ \\
Enurese na infância & $12(57,1)$ \\
Atividade física &
\end{tabular}

O fator de risco apresentado como dominante foi o consumo de alimentos irritativos (90\%) sendo o café (70\%) o mais prevalente, seguido de alimentos cítricos (55\%) e alimentos apimentados (30\%). Outros fatores de risco destacaram-se como parto normal (60\%) com episiotomia (65\%), cesariana (30\%) e história de gestações, com mediana de 2,0 (1,0-3,5), dados demonstrados na Tabela 2.

Tabela 2. Descrição dos fatores de risco associados à IU em 28 participantes com queixa clínica de incontinência urinária de esforço. Salvador, 2018

\begin{tabular}{lc}
\hline Variáveis & Resultados \\
\hline Gestação* & $2,0(1,0-3,5)$ \\
Parto* & $2,0(1,0-3,0)$ \\
Aborto * & $0,0(0,0-1,0)$ \\
\hline Tipo de parto & $\mathbf{n}(\%)$ \\
Normal & $12(60,0)$ \\
Cesário & $06(30,0)$ \\
Misto & $02(10,0)$ \\
Episiotomia & $13(65,0)$ \\
\hline Alimentos irritativos & \\
Sim & $18(90,0)$ \\
Não & $02(10,0)$ \\
\hline Tipos de alimentos & \\
Café & $14(70,0)$ \\
Cítricas & $11(55,0)$ \\
Chá preto & $02(10,0)$ \\
Carbonadas & $05(25,0)$ \\
Apimentadas & $06(30,0)$ \\
\hline Chocolate & $05(25,0)$ \\
\hline
\end{tabular}


As queixas clínicas mais prevalentes foram: perda ao tossir (96,3\%) e ao espirrar (92,6\%), seguidos por sensação de resíduo $(81,5 \%)$ e perda ao realizar atividade física $(70,4 \%)$. A perda ao esforço foi relatada como "sempre" em $57,1 \%$ dos casos. $O$ uso do forro foi apresentado por $60 \%$ das mulheres e a menopausa foi relatada por $52,4 \%$ (Tabela 3).

Tabela 3. Descrição dos sintomas clínicos das 28 participantes com IUE. Salvador, 2018

\begin{tabular}{lc}
\hline Variáveis & Resultados \\
\hline Sintomas & $\mathbf{n}(\%)$ \\
Disúria & $01(4,8)$ \\
Sensação de resíduo & $22(81,5)$ \\
Desejo pós-miccional & $15(55,6)$ \\
Flatus vaginais & $13(61,9)$ \\
Perda ao esforço & $27(100,0)$ \\
Frequência de perda ao esforço & \\
Sempre & $12(57,1)$ \\
As vezes & $07(25,0)$ \\
Raramente & $02(9,5)$ \\
Momento da perda & \\
Tosse & $26(96,3)$ \\
Espirro & $25(92,6)$ \\
Durante a Atividade física & $19(70,4)$ \\
Riso & $17(63,0)$ \\
Corrida & $14(51,9)$ \\
Caminhar & $11(40,7)$ \\
Relação sexual & $10(37,0)$ \\
Uudança de posição & $12(60,0)$ \\
Mena forro & $11(52,4)$ \\
Reposição hormonal & $04(19,0)$ \\
Sensação de peso vaginal & $05(23,8)$ \\
\hline & $08(38,1)$ \\
\hline
\end{tabular}

As cirurgias relatadas foram: 01 desobstrução de trompas (3,6\%), 01 colpoperineoplastia (3,6\%), 04 histerectomias (14,3\%), 01 plástica vaginal (3,6\%). O Pad Test teve maior prevalência de perda leve $(57,1 \%)$, seguido por perda moderada (39,3\%) e perda grave (3,6\%), como no Gráfico 1. 


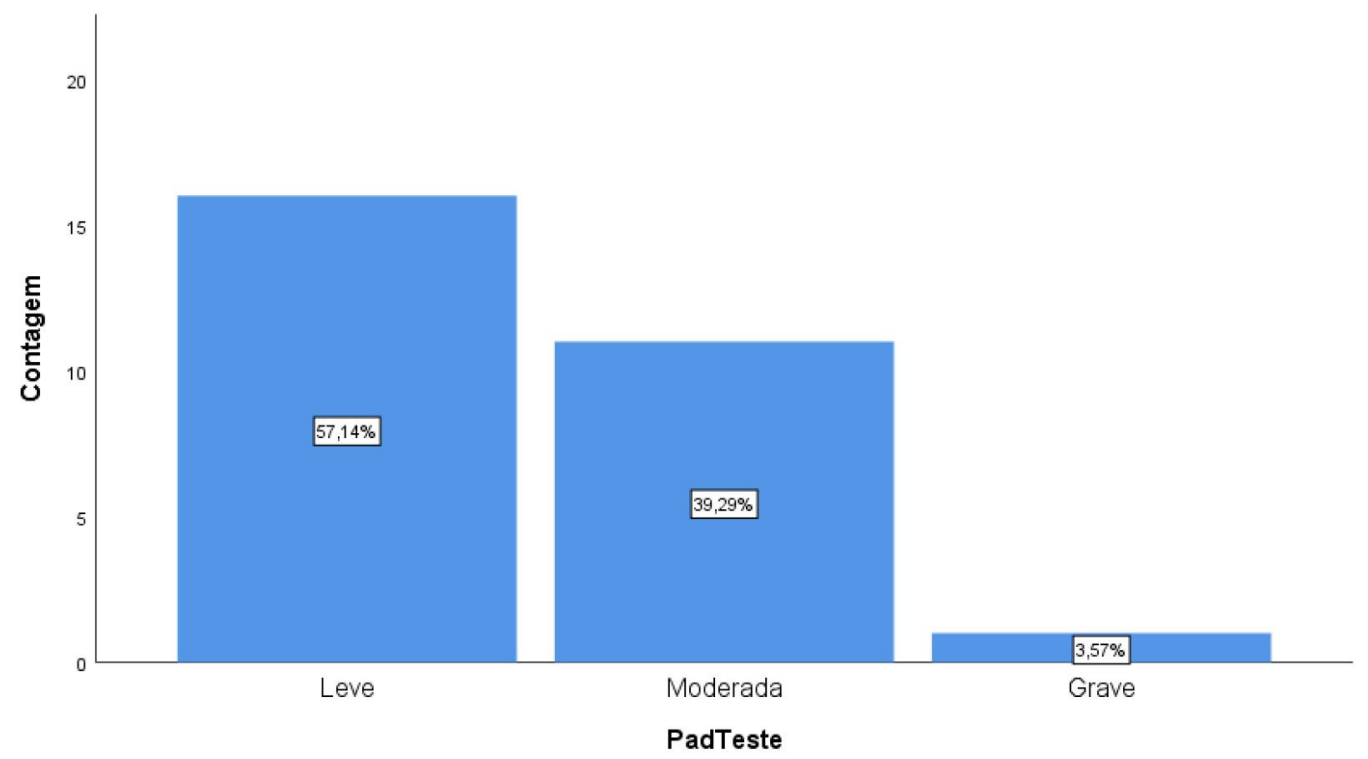

As variáveis obtidas através do diário miccional tiveram como frequência miccional mediana de $7,0(5,9$ - 6,2), capacidade máxima urinada 300,0 (200,0 - 428,5), ingesta hídrica $1.584(1.347$ - 2330) e perda urinária 2,3 $(0,7$ - 4,0), demonstradas na Tabela 4.

Tabela 4. Descrição das variáveis obtidas através do diário miccional de 28 participantes com queixa clínica de incontinência urinária de esforço. Salvador, 2018

\begin{tabular}{lc}
\hline Variáveis & $\begin{array}{c}\text { Mediana (intervalo } \\
\text { interquartil) }\end{array}$ \\
\hline Tempo de sintoma & $36,0(6,0-60,0)$ \\
Frequência miccional & $7,0(5,9-9,2)$ \\
Capacidade máxima & $300,0(200,0-428,5)$ \\
Capacidade média & $224,0(174,0-282,0)$ \\
Intervalo miccional & $156,0(123,5-195,5)$ \\
Ingesta hídrica & $1.584(1.347-2.330)$ \\
Perda urinária & $2,3(0,7-4,0)$ \\
\hline
\end{tabular}

\section{Discussão}

A análise da incontinência urinária e os fatores associados são relevantes não somente por considerar como um grave problema de saúde pública, mas também pelo impacto negativo na vida das mulheres que possuem IUE.6-8 No presente estudo, encontrada maior prevalência em mulheres de meia idade, o que corrobora com outros estudos que afirmam que mulheres acometidas pela IUE apresentam faixa etária mais prevalente acima dos 40 anos, e a prevalência se mantém crescente. $\frac{15,16}{16}$

Os fatores de risco para IUE foram a ingestão de cafeína, alimentos cítricos, alimentos apimentados, parto normal com episiotomia e cesariana. O parto normal pode apresentar maior quantidade de traumas no assoalho pélvico, aumentando o risco para IUE. ${ }^{17}$ Apesar da ingestão de cafeína estar relacionada com a IUU, devido à instabilidade que a cafeína causa no músculo detrusor, percebemos que neste estudo também foi prevalente, mesmo em pacientes com IUE. ${ }^{18}$ Este dado pode refletir a presença de mulheres com incontinência urinária mista, que não foram excluídas do estudo. 
O sintoma relatado como mais prevalente foi a sensação de resíduo e os momentos da perda urinária mais frequentes foram ao realizar esforço físico importantes como tossir, espirrar ou praticar atividade física. Por se tratar de IUE, era esperado que as participantes relatassem perda urinaria no momento de aumento da pressão abdominal, justificado pela fisiopatologia da IUE. Referente à sensação de resíduos, pode haver duas explicações, uma limitação de não avaliar se de fato as participantes apresentavam instabilidade vesical ou até mesmo um viés de relato, visto que não foi realizado USG de resíduo pós-miccional. Na literatura encontramos como as principais queixas relacionadas à IUE condizentes com o estudo abordado. $\frac{15}{5}$

As comorbidades associadas mais prevalentes foram obesidade, hipertensão, constipação e menopausa, muitas já relatadas na literatura..$^{19}$ Estudos mostram que o IMC elevado aumenta o risco de IUE, devido à maior pressão sobre a musculatura pélvica, prejudicando a funcionalidade muscular. 20 A menopausa pode estar relacionada à IUE devido às alterações hormonais que afetam a musculatura pélvica. ${ }^{21}$ Mulheres hipertensas são mais propensas à IUE, provavelmente devido ao uso de diuréticos. Este dado encontrado é importante, pois poderia guiar uma mudança terapêutica, a fim de melhorar a qualidade de vida das mulheres sintomáticas. ${ }^{22}$ A constipação pode estar relacionada com a IUE devido à pressão constante realizada sobre a musculatura pélvica para inibir a perda urinária e ocasionar uma dificuldade de defecar. $\underline{\underline{23}}$

Quanto ao Pad Test, encontrada maior prevalência de incontinência urinária leve $(57,14 \%)$, seguido de moderada (39,29\%). De acordo com a literatura, a prevalência de IUE leve é maior, chegando a 75\%, seguido de moderada (20\%). $\frac{25}{}$ Os dados encontrados no Pad Test foram compatíveis com as queixas das participantes, já que apresentavam prevalência de perdas com esforço importante, comuns em perdas leves. Não houve relato de perda com esforços leves, a exemplo de mudança de decúbito, que seria esperado em incontinências graves.

A mediana encontrada no diário miccional da capacidade média miccional foi de 224 (174 -282), a frequência miccional $7(5,9-9,2)$, capacidade máxima eliminada $300(200$ - 428,5), perda urinária 2,3 (0,7 $4,0)$. Na literatura a média apresentada como normal da capacidade média miccional é entre 230-250, a frequência miccional 5,7-7,3, capacidade máxima eliminada 1.272-1350. $\frac{25}{}$ Diante dos resultados, as participantes apresentaram disfunção de armazenamento e esvaziamento da bexiga, o que pode ser a causa da incontinência urinária. ${ }^{26} \mathrm{~A}$ perda urinária encontrada no diário miccional confirma a incontinência urinária relatada pelas participantes do estudo.

O presente estudo apresentou como limitações o pequeno tamanho amostral que possibilite análises estatísticas e extrapolação dos seus resultados. Sugere-se, para descrição mais fidedigna, a realização de estudos multicêntricos, com maior número de participantes.

\section{Conclusão}

Dentre as mulheres com incontinência urinária de esforço avaliadas no período do estudo, destaca-se como fatores mais frequentes a idade avançada, o status menopausal, obesidade, hipertensão, multíparas e que realizaram parto vaginal com episiotomia, constipação, e aquelas que ingerem cafeína. Houve uma maior prevalência de incontinência urinária leve.

\section{Contribuições dos autores}

Lordelo P contribuiu com o desenho do estudo. Brasil C e Lemos A realizaram análise dos dados. Machado $\mathrm{M}$ e Moura T contribuíram com a coleta e interpretação dos dados. Alves R contribuiu com a redação do manuscrito. Todos os autores participaram da aprovação da versão final do manuscrito.

\section{Conflitos de interesses}

Nenhum conflito financeiro, legal ou político envolvendo terceiros (governo, empresas e fundações privadas, etc.) foi declarado para nenhum aspecto do trabalho submetido (incluindo, mas não se limitando a subvenções e financiamentos, participação em conselho consultivo, desenho de estudo, preparação de manuscrito, análise estatística, etc.). 


\section{Referências}

1. Haylen BT, Ridder D, Freeman RM, Swift SE, Berghmans $B$, Lee J, et al. An International Urogynecological Association (IUGA)/International Continence Society (ICS) joint report on the terminology for female pelvic floor dysfunction. Int Urogynecol J. 2010;21(1):5-26. https://doi.org/10.1007/s00192-009-0976-9

2. Santos CRS, Santos VLG. Prevalência da incontinência urinária em amostra randomizada da população urbana de Pouso Alegre, Minas Gerais, Brasil. Rev. Latino-Am. Enf. 2010;18(5):903-10. https://doi.org/10.1590/S0104-11692010000500010

3. Siddiqui NY, Levin PJ, Phadtare A, Pietrobon R, Ammarell N. Perceptions about female urinary incontinence: a systematic review. Int Urogynecol J. 2014;25(7):863-71. https://doi. org/10.1007/s00192-013-2276-7

4. Ebbesen MH, Hunskaar S, Rortveit G, Hannestad YS. Prevalence, incidence and remission of urinary incontinence in women: longitudinal data from the Norwegian HUNT study (EPINCONT). BMC Urol. 2013;13:27. https://doi.org/10.1186/1471-2490-13-27

5. Townsend MK, Danforth KN, Lifford KL, Rosner B, Curhan GC, Resnick NM, et al. Incidence and remission of urinary incontinence in middle-aged women. Am J Obstet Gynecol. 2007;197(2):167. e1-5. https://doi.org/10.1016/j.ajog.2007.03.041

6. Carvalho MP, Andrade FP, Peres W, Martinelli T, Simch $\mathrm{F}$, Orcy RB, et al. O impacto da incontinência urinária e seus fatores associados em idosas. Rev. Bras. Geriatr. Gerontol. 2014;17(4):721-30. http://dx.doi.org/10.1590/18099823.2014.13135

7. Lalos O, Berglund AL, Lalos A. Impact of urinary and climacteric symptoms on social and sexual life after surgical treatment of stress urinary incontinence in women: a long-term outcome. J Adv Nurs. 2001;33(3):316-27. https://doi.org/10.1046/j.13652648.2001.01667.x

8. Swithinbank LV, Abrams P. The impact of urinary incontinence on the quality of life of women. World J Urol. 1999;17(4):225-9. https://doi.org/10.1007/s003450050137

9. Grzybowska ME, Wydra D. 24/7 usage of continence pads and quality of life impairment in women with urinary incontinence. Int J Clin Pract. 2019;73(8):e13267. https://doi.org/10.1111/ijcp.13267

10. Bump RC, Norton PA. Epidemiology and natural history of pelvic floor dysfunction. Obstet Gynecol Clin North Am. 1998;25(4):723-46. https://doi.org/10.1016/s0889-8545(05)70039-5

11. Silva JCP, Soler ZASG, Wysocki AD. Fatores associados à incontinência urinária em mulheres submetidas ao exame urodinâmico. Rev esc. enf. USP. 2017;51:e03209. http://dx.doi. org/10.1590/s1980-220×2016140903209
12. Delancey JO, Ashton-Miller JA. Pathophysiology of adult urinary incontinence. Gastroenterology. 2004;126(1 Supl 1):S23-32. https://doi.org/10.1053/j.gastro.2003.10.080

13. Butler RN, Maby Jl, Montella JM, Young GP. Urinary incontinence: keys to diagnosis of the older woman.1. Geriatrics. 1999;54(10):22-6, 29-30. Citado em: PMID: 10542858

14. Agur W, Housami F, Drake M, Abrams P. Could the National Institute for Health and Clinical Excellence guidelines on urodynamics in urinary incontinence put some women at risk of a bad outcome from stress incontinence surgery? BJU Int. 2009;103(5):635-9. https://doi.org/10.1111/j.1464410x.2008.08121.x

15. Abrams P, Cardozo L, Fall M, Griffiths D, Rosier P, Ulmsten U, et al. The standardisation of terminology in lower urinary tract function: report from the standardisation sub-committee of the International Continence Society. Urology. 2003;61(1):37-49. https://doi.org/10.1016/S0090-4295(02)02243-4

16. Hannestad YS, Rortveit G, Sandvik H, Hunskaar S; Norwegian EPINCONT study. Epidemiology of Incontinence in the County of Nord-Trøndelag. A community-based epidemiological survey of female urinary incontinence: the Norwegian EPINCONT study. Epidemiology of Incontinence in the County of Nord-Trøndelag. J Clin Epidemiol. 2000;53(11):1150-7. https://doi.org/10.1016/s08954356(00)00232-8

17. Rortveit G, Daltveit AK, Hannestad YS, Hunskaar S. Urinary incontinence after vaginal delivery or cesarean section. $\mathrm{N}$ Engl J Med. 2003;348:900-7. http://dx.doi.org/10.1056/NEJMoa021788

18. Marques LP, Schneider IJC, Giehl MWC, Antes DL, d'Orsi E. Fatores demográficos, condições de saúde e hábitos de vida associados à incontinência urinária em idosos de Florianópolis, Santa Catarina. Rev Bras Epidemiol. 2015;18(3):595-606. http:// dx.doi.org/10.1590/1980-5497201500030006

19. Higa R, Lopes MHBM, Reis MJ. Fatores de risco para incontinência urinária na mulher. Rev. Esc. Enf. USP. 2008;42(1):187-92. http://dx.doi.org/10.1590/S0080$\underline{62342008000100025}$

20. Lyu XL, Xu B, Song J, Zhong XL, Song YF. Prediction of pelvic floor muscle function in stress urinary incontinence. Zhonghua Fu Chan Ke Za Zhi. 2018;53(10):694-9. https://doi.org/10.3760/ cma.j.issn.0529-567x.2018.10.008

21. Sacomori C, Negri NB, Cardoso FL. Incontinência urinária em mulheres que buscam exame preventivo de câncer de colo uterino: fatores sociodemográficos e comportamentais. Cad. Saúde Pública. 2013;29(6):1251-9. https://doi.org/10.1590/S0102$311 \times 2013000600021$ 
22. Berlezi EM, Fiorin AAM, Bilibio PVF, Kirchner RM, Oliveira KR. Estudo da incontinência urinária em mulheres climatéricas usuárias e não usuárias de medicação anti-hipertensiva. Rev. Bras. Geriatr. Geront. 2011;14(3):415-23. https://doi.org/10.1590/ S1809-98232011000300002

23. Cameron AP, Smith AR, Lai HH, Bradley CS, Liu AB, Merion RM, et al. Bowel function, sexual function, and symptoms of pelvic organ prolapse in women with and without urinary incontinence. Neurourol Urodyn. 2018;37(8):2586-96. https://doi.org/10.1002/ nau. 23587

24. Yang JM, Yang SH, Yang SY, Yang E, Huang WC, Tzeng CR. Clinical and pathophysiological correlates of the symptom severity of stress urinary incontinence. Int Urogynecol J. 2010;21(6):637-43. https://doi.org/10.1007/s00192-009-1094-4
25. Fitzgerald MP, Stablein U, Brubaker L. Urinary habits among asymptomatic women. Am J Obstet Gynecol. 2002;187(5):1384-8. https://doi.org/10.1067/mob.2002.126865

26. Lucas MG, Bosch JLHR, Cruz F, Rider DJMK, Madden TB, Nambiar A, et al. Diretrizes para incontinência urinária. Portal da Urologia [Internet]; 2012. Disponível em: http://portaldaurologia. org.br/medicos/wp-content/uploads/2017/06/223.pdf 